\title{
EFEITOS DE REGULADORES DE CRESCIMENTO NA FRUTIFICAÇÃO DO MORANGUEIRO CULTIVAR «MONTE ALEGRE»*
}

\author{
Paulo R. C. Castro** \\ KEIGo MiNAMI*** \\ Natal A. Vello****
}

\section{RESUMO}

Estudou-se em Piracicaba (SP), os efeitos da aplicação de reguladores de crescimento em morangueiro cultivar "Monte Alegre". Efetuou-se a pulverização de SADH 5000 ppm, CCC 2000 ppm e GA $550 \mathrm{ppm}$; sendo que realizaram-se ainda três aplicações de IAA $10 \mathrm{ppm}$ e GA $10 \mathrm{ppm}$, além do tratamento controle. Verificou-se que apesar do GA 550 ppm, SADH e CCC reduzirem a produção, três aplicações de IAA $10 \mathrm{ppm}$ ou GA $10 \mathrm{ppm}$ promoveram uma tendência de aumento na produtividade do morangueiro. Notou-se que os reguladores de crescimento não alteraram o número de frutos; sendo que o tratamento com GA 550 ppm promoveu redução no peso médio dos frutos da cultivar "Monte Alegre". Aplicação de GA $550 \mathrm{ppm}$ causou aumento no número de frutos na terceira e quarta semanas de colheita, reduzindo progressivamente nas semanas subseqüentes. Observou-se que aplicações de GA $10 \mathrm{ppm}$ ou IAA $10 \mathrm{ppm}$ promoveram aumento no comprimento dos pedúnculos, facilitando a colheita.

\section{INTRODUÇÃO}

O conhecimento dos efeitos de reguladores de crescimento em morangueiro reveste-se de interesse, pois a aplicação desses produtos químicos pode melhorar quantitativa e qualitativamente a frutificação do morangueiro.

Apesar de poucos estudos terem sido efetuados sobre a ação de fitohormônios em Fragaria sp., a crescente importância econômica da

\footnotetext{
* Entregue para publicação em 15/3/1976.

* Departamento de Botânica. E.S.A. "Luiz de Queiroz" - USP.

*** Departamento de Agricultura e Horticultura. E.S.A. "Luiz de Queiroz" - USP.

*** Departamento de Genética. E.S.A. "Luiz de Queiroz" - USP.

OBS. Considerar aspas das cultivares como sendo aspas simples
} 
cultura e a utilização de técnicas avançadas de cultivo, implica na necessidade destes estudos.

Os reguladores de crescimento têm sido utilizados principalmente para obter-se aumentos no tamanho dos frutos e na colheita; além de promover precocidade ou atraso no florescimento e maturação dos frutos do morangueiro (WEAVER, 1972).

A cultivar "Monte Alegre", juntamente com a cultivar "Campinas", tem demonstrado possuir precocidade, produtividade e características comerciais do produto, significativamente superiores às demais cultivares, nas condições das principais regiões de cultivo do Estado de São Paulo (CAMARGO et alii, 1974).

O presente trabalho teve como finalidade verificar os efeitos da aplicação de reguladores de crescimento na frutificação do morangueiro cultivar "Monte Alegre".

\section{REVISÃO DA LITERATURA}

BERGAMINI \& PIMPINI (1968) efetuaram três pulverizações em morangueiros, durante o florescimento, com SADH (ácido succínico - 2,2 - dimetilhidrazida) nas concentrações de 500 a 8000 ppm. Dosagens acima de 2000 ppm promoveram efeitos adversos nas dimensões dos frutos, na colheita e na resistência à infecção fúngica. A porcentagem de frutos não comercializáveis aumentou com aplicações de dosagens acima de 1000 ppm. Não se observaram efeitos significativos na época de maturação, peso dos frutos, textura ou no conteúdo de açúcar. $O$ número de estolhos por planta e o comprimento dos mesmos, foi reduzido com aplicações de SADH 4000 ppm e doses superiores. Concentrações acima de 500 ppm reduziram o comprimento do pecíolo e a área foliar, aumentando a espessura das folhas.

PUFFER et alii (1968) observou que o SADH pode aumentar a produtividade do morangueiro, sob determinadas condições. O regulador de crescimento parece tornar as plantas mais tolerantes à seca e afetar a precocidade de produção.

FREEMAN \& CARNE (1970) encontraram evidências de que a requeima pelo frio em plantas de morangueiro "Northwest" pode ser reduzida pelo SADH. Verificaram que a concentração de 5000 ppm mostrou-se mais adequada; sendo que consideraram que o SADH promove uma proteção dos botões florais.

GUERRIERO et alii (1969) observaram que aplicação de cloreto de (2-cloroetil) trimetilamônio (CCC) em morangueiro, pode controlar o desenvolvimento dos estolhos; sendo que o tratamento reduziu o número e o comprimento dos estolhos secundários. Aplicações de 3 a $4 \%$ de CCC promoveram as melhores respostas na produção. 
LORETI \& VITAGLIANO (1970) verificaram que o CCC $3 \%$ aumentou a produtividade das cultivares "Cambridge Vigour" $\mathrm{e}$ "Oranda", de morangueiro. Dosagens de 4 a 5\% de CCC promoveram efeitos não significativos ou depressivos, na produção.

DENNIS \& BENNETT (1969) observaram que aplicação de GA (ácido giberélico) $550 \mathrm{ppm}$, em morangueiro, estimulou a produção de estolhos.

HONDA (1972) verificou que pulverização com GA promoveu aumento no número de estolhos do morangueiro. A aplicaçã̃o de GA, 30 dias antes do florescimento, causou precocidade de duas semanas no aparecimento das últimas flores. Aplicação de GA durante a antese floral promoveu precocidade na maturação dos frutos e aumento no comprimento dos pecíolos e pedúnculos.

SOLOVEI (1972) observou que tratamento de morangueiro com GA nas dosagens de 25 e $50 \mathrm{ppm}$ aumentou o número de estolhos e de folhas por planta. Concentrações de 100 e $200 \mathrm{ppm}$ de GA promoveram inibição no desenvolvimento dos estolhos.

PATTLAK \& SINGH (1971) notaram precocidade de 7 a 17 dias no florescimento e de 7 a 25 dias na colheita do morangueiro, com aplicação de GA $75 \mathrm{ppm}$. O produto causou também aumento na elongação do pedúnculo e no número de frutos por planta.

TAVAOZE \& MAZANASHVILI (1973) observaram que o tratamento de morangueiros nos estágios iniciais de desenvolvimento, com GA, aumentou o crescimento e o florescimento. Na maioria dos casos o GA reduziu as produções, mas melhorou substancialmente a qualidade dos frutos, aumentando os teores de vitamina $\mathrm{C}$ e açúcar.

SMITH et alii (1961) verificaram que os melhores resultados no aumento da produção de frutos de morangueiro, nos primeiros estágios da colheita, foram obtidos com três aplicações de GA 10 ppm, em intervalos semanais, no início do florescimento. Observaram maior produção de flores no início do período de florescimento, promovendo conseqüentemente precocidade na colheita da cultivar "Sparkle". Notaram que apesar do acentuado aumento de produção nas três primeiras colheitas, a produtividade final mostrou-se semelhante. Na terceira colheita, observaram que o peso dos frutos tratados revelou-se ocasionalmente inferior ao controle, mas não ocorreu diferença no tamanho dos frutos, considerando-se a totalidade das colheitas.

\section{MATERIAIS E MÉTODOS}

Iniciou-se o experimento em 24 de abril de 1975, em Piracicaba (SP), tendo-se efetuado o transplante de mudas uniformes do moran- 
gueiro cultivar "Monte Alegre" para 5 canteiros de 20 metros, com duas linhas cada um.

O solo utilizado trata-se de uma Terra Roxa Estruturada série Luiz de Queiroz.

O delineamento experimental utilizado foi em blocos ao acaso, tendo-se 6 tratamentos em 5 blocos, sendo cada parcela constituida de 15 plantas.

Os tratamentos experimentais constaram, além do controle (T1), da aplicação, sob a forma de pulverização em 16/05/75 de SADH 5000 ppm (T2), CCC $2000 \mathrm{ppm}$ (T3) e GA $550 \mathrm{ppm}$ (T6). Aplicações de IAA (ácido indolacético) -T4 e GA na concentração de $10 \mathrm{ppm}$ (T5), foram realizadas nos dias 16, 23 e 30/05/75. Em todas as pulverizagóes utilizaram-se 3 litros de solução.

Efetuou-se a cobertura do solo com serragem.

As colheitas foram iniciadas em $08 / 07 / 75$ e finalizadas em $12 / 09 / 75$, tendo sido efetuadas duas vezes por semana. Verificou-se o número de frutos colhidos por parcela de 15 plantas, sendo que o peso dos mesmos foi determinado em balança Mettler P1200N. Verificou-se também o peso médio por fruto, além de observar-se algumas variações no desenvolvimento das plantas.

Analisaram-se estatisticamente os parâmetros referentes a quatro etapas de colheita, de $08 / 07$ a $22 / 07 / 75$, de $25 / 07$ a $08 / 08 / 75$, de $12 / 08$ a 26/08/75 e de 29/08 a 12/09/75. Efetuaram-se ainda as análises de regressão de cada tratamento sobre as quatro etapas de colheita.

\section{RESULTADOS}

Na tabela 1 observamos a ocorrência de diferenças significativas entre os tratamentos, no que se refere ao peso e ao peso médio dos frutos de morangueiro. Isto demonstra que os reguladores de crescimento afetaram a frutificação do morangueiro cultivar "Monte Alegre". Verificamos também variações nas etapas de colheita e na relação entre etapas e tratamentos, quanto à produção, número e peso médio dos frutos colhidos.

A tabela 2 mostra as médias gerais para etapas de colheita, dos parâmetros estudados, sob efeito dos tratamentos realizados. Observamos, pelo teste de Tukey, que os tratamentos com GA $550 \mathrm{ppm}$, SADH 5000 ppm e CCC 2000 ppm, promoveram diminuição na produção do morangueiro. Apesar dos tratamentos IAA $10 \mathrm{ppm}$ ( 3 apl.) e GA $10 \mathrm{ppm}$ (3 apl.) mostrarem uma tendência de aumento na produção, 
Tabela 1 - Análises de variância dos caracteres: produção (gramas), número de frutos transformado em $\log (X+1)$ e peso médio de fruto de morangueiro em gramas. Delineamento em blocos ao acaso com parcelas subdivididas no tempo.

\begin{tabular}{|c|c|c|c|c|}
\hline \multirow{2}{*}{ F.V. } & \multirow{2}{*}{ G.L. } & \multicolumn{3}{|c|}{ Q.M. } \\
\hline & & Produção & $\mathrm{N} .{ }^{\circ}$ frutos & Peso médio \\
\hline Blocos (B) & 4 & 154595,8 & 0,026586 & 2,7515 \\
\hline Tratamentos $(\mathrm{T})$ & 5 & $859874,0^{* *}$ & 0,073467 & $33,5533^{* *}$ \\
\hline Resíduo a & 20 & 147651,0 & 0,048569 & 3,6735 \\
\hline Etapas (E) & 3 & $4618553,2^{* *}$ & $2,926261 * *$ & $338,5097 * *$ \\
\hline $\mathrm{E} \times \mathrm{T}$ & 15 & $347062,1 * *$ & $0,143197 * *$ & $8,5987 * *$ \\
\hline $\mathrm{E} \times \mathrm{B}$ & 12 & 56557,8 & 0,041583 & 1,6198 \\
\hline $\mathrm{E} \times \mathrm{T} \times \mathrm{B}$ & 60 & 73254,1 & 0,036496 & 1,6947 \\
\hline (Resícluo b) & (72) & 70471,4 & 0,037344 & 1,6822 \\
\hline \multicolumn{5}{|l|}{ Total } \\
\hline $\mathrm{X}$ & & 1174,8 & 2,103994 & 8,66 \\
\hline C.V. (a) & & $32,7 \%$ & $10,5 \%$ & $22,1 \%$ \\
\hline C.V. (b) & & $22,6 \%$ & $9,2 \%$ & $15,0 \%$ \\
\hline
\end{tabular}

** significativo ao nível de $1 \%$ de probabilidade

não diferiram significativamente do controle. Considerando-se as 4 etapas analisadas isoladamente, notamos que nas duas primeiras etapas de colheita não ocorreram diferenças significativas na produção; na terceira etapa os tratamentos GA $10 \mathrm{ppm}$ e IAA $10 \mathrm{ppm}$ mostraram produções significativamente mais altas e o tratamento GA $550 \mathrm{ppm}$ apresentou peso dos frutos significativamente inferior; na quarta etapa apenas GA $550 \mathrm{ppm}$ reduziu a produção em relação aos demais tratamentos.

Observamos na tabela 2 que os tratamentos com reguladores de crescimento não afetaram o número de frutos colhidos, considerando-se as médias gerais para etapas de colheita. As etapas analisadas sepa- 
radamente apresentaram, pelo teste de Tukey, variações significativas no número de frutos, na primeira e quarta etapas. $\mathrm{Na}$ primeira etapa de colheita o tratamento com SADH 5000 ppm reduziu o número de frutos, sendo que GA $550 \mathrm{ppm}$ aumentou o número de frutos colhidos. $\mathrm{Na}$ quarta etapa de colheita o tratamento com GA $550 \mathrm{ppm}$ reduziu significativamente o número de frutos colhidos, em relação aos demais tratamentos.

Tabela 2 - Médias gerais para etapas de colheita, no que se refere à produção em gramas e número de frutos por 15 plantas, além do peso médio de frutos de morangueiros em gramas, submetidos a seis tratamentos.

\begin{tabular}{|c|c|c|c|}
\hline Tratamento & Produção & Número & Peso médio \\
\hline Controle & $1264,54 \mathrm{a}$ & 165,65 a & $9,766 \mathbf{a}$ \\
\hline SADH $5000 \mathrm{ppm}$ & $1081,06 \mathrm{a} \mathrm{b}$ & $152,50 \mathrm{a}$ & $9,288 \mathbf{a}$ \\
\hline CCC $2000 \mathrm{ppm}$ & 1164,31 a b & 145,95 a & $9,109 \mathbf{a}$ \\
\hline IAA $10 \mathrm{ppm}$ (3 apl.) & $1397,86 \mathrm{a}$ & $175,30 \mathrm{a}$ & $9,186 \mathbf{a}$ \\
\hline GA 10 ppm (3 apl.) & $1321,88 \mathrm{a}$ & $179,30 \mathrm{a}$ & $8,463 \mathbf{a}$ \\
\hline GA $550 \mathrm{ppm}$ & $818,95 \mathrm{~b}$ & $133,80 \mathrm{a}$ & $6,159 \mathrm{~b}$ \\
\hline
\end{tabular}

obs.: valores seguidos da mesma letra não diferem entre si, pelo teste de Tukey.

Tabela 3 - Médias gerais para tratamentos, no que se refere à produção em gramas e número de frutos por 15 plantas, além do peso médio de frutos de moran. gueiros em gramas, em quatro etapas de colheita.

\begin{tabular}{rrrr}
\hline \multicolumn{1}{c}{ Etapa } & Produção & Número & Peso médio \\
\hline & & & \\
$08 / 07-22 / 07$ & $637,25 \mathrm{a}$ & $51,97 \mathrm{a}$ & $13,355 \mathrm{a}$ \\
$25 / 07-08 / 08$ & $1313,34 \mathrm{~b}$ & $161,07 \mathrm{~b}$ & $8,423 \mathrm{~b}$ \\
$12 / 08-26 / 08$ & $1566,78 \mathrm{c}$ & $208,20 \mathrm{~b}$ & $7,395 \mathrm{c}$ \\
$29 / 08-12 / 09$ & $1181,69 \mathrm{~b}$ & $213,77 \mathrm{~b}$ & $5,473 \mathrm{~d}$ \\
& & & \\
\hline
\end{tabular}

obs.: valores seguidos da mesma letra não diferem entre si, pelo teste de Tukey. 
Tabela 4 - Análises de regressão de cada tratamento sobre quatro etapas de colheita, para os caracteres: produção (gramas) e número de frutos por 15 plantas transformado em $\log (X+1)$ e peso médio de fruto de morangueiro em gramas.

\begin{tabular}{|c|c|c|c|c|}
\hline \multirow{2}{*}{ F.V. } & \multirow{2}{*}{ G.L. } & \multicolumn{3}{|c|}{ Q.M. } \\
\hline & & Produção & $n^{0}$ frutos & Peso médio \\
\hline RL (T 1) & 1 & $1646319,9 * *$ & $1,754806^{* *}$ & $297,8731^{* *}$ \\
\hline $\mathrm{RQ}(\mathrm{T} 1)$ & 1 & $889871,5^{* *}$ & $0,377306^{* *}$ & $41,1558^{* *}$ \\
\hline $\mathrm{RC}(\mathrm{T} 1)$ & 1 & 52271,7 & 0,082876 & $12,8379^{* *}$ \\
\hline RL ( ( $\mathrm{T}$ 2) & 1 & $2122761,7 * *$ & $3,042752^{* *}$ & $274,7638^{* *}$ \\
\hline $\mathrm{RQ} \quad\left(\begin{array}{ll}\mathrm{T} & 2\end{array}\right)$ & 1 & $1105863,4^{* *}$ & $0,612460^{* *}$ & $15,0685^{* *}$ \\
\hline $\mathrm{RC}(\mathrm{T} 2)$ & 1 & 35717,2 & 0,036229 & 0,1076 \\
\hline RL ( $\left.\begin{array}{ll}\mathrm{T} & 3\end{array}\right)$ & 1 & $885368,1^{* *}$ & $0,907893^{* *}$ & $140,8257 * *$ \\
\hline $\mathrm{RQ}(\mathrm{T} 3)$ & 1 & $1362420,0^{* *}$ & $0,332554 * *$ & $13,0088^{* *}$ \\
\hline $\mathrm{RC}(\mathrm{T} \quad 3)$ & 1 & 14380,8 & 0,040487 & $10,1697^{*}$ \\
\hline RL ( $(\mathrm{T}$ 4) & 1 & $1437001,6 * *$ & $1,02258()^{* *}$ & $155,4510^{* *}$ \\
\hline $\mathrm{RQ}(\mathrm{T} 4)$ & 1 & $1356111,1^{* *}$ & $0,194173^{*}$ & $10,1674^{*}$ \\
\hline $\mathrm{RC}(\mathrm{T} \quad 4)$ & 1 & $320299,4^{*}$ & 0,001335 & $12,1522^{* *}$ \\
\hline RL ( $\mathrm{T} 5$ ) & 1 & $1718773,4^{* *}$ & $1,366424^{* *}$ & $177,4839^{* *}$ \\
\hline $\mathrm{RQ}(\mathrm{T} 5)$ & 1 & $2523767,1 * *$ & $0,464901 * *$ & $6,8328^{*}$ \\
\hline $\mathrm{RC}\left(\begin{array}{ll}\mathrm{T} & 5\end{array}\right)$ & 1 & $1289496,5^{* *}$ & 0,002712 & 9,3208* \\
\hline RL ( $\left(\begin{array}{l}\text { T } \\
\text { ) }\end{array}\right.$ & 1 & $281377,2^{*}$ & 0,015418 & $25,0801 * *$ \\
\hline $\mathrm{RQ}(\mathrm{T}$ 6) & 1 & $1448027,1^{* *}$ & $0,540804^{* *}$ & 0,2333 \\
\hline $\mathrm{RC}\left(\begin{array}{ll}\mathrm{T} & 6\end{array}\right)$ & 1 & $571762,8^{* *}$ & 0,131034 & 1,9768 \\
\hline Resíduo b & 72 & 70471,4 & 0,037344 & 1,6822 \\
\hline
\end{tabular}

* significativo ao nível de $5 \%$ de probabilidade

** significativo ao nível de $1 \%$ de probabilidade

Pela tabela 2 notamos que o tratamento com GA $550 \mathrm{ppm}$ reduziu significativamente o peso médio dos frutos. Análise isolada das etapas de colheita, mostrou diminuição no peso médio dos frutos, com aplicação de GA 550 ppm, em todas as etapas; sendo porém que diminuições significativas, em relação ao controle, foram notadas somente na primeira e segunda etapas.

A tabela 3 apresenta as médias gerais para tratamentos, nas quatro etapas de colheita. Verificamos, pelo teste de Tukey, que a produção mostrou-se significativamente mais elevada na terceira etapa de colheita, média na segunda e quarta etapas, e revelou-se inferior na primeira 
etapa. Considerando-se o efeito dos tratamentos isoladamente, nas quatro etapas, observamos que todos os tratamentos produziram menos na primeira etapa. Os tratamentos IAA $10 \mathrm{ppm}$ (3 apl.) e GA 10 ppm (3 apl.) promoveram aumentos significativos em produção, na terceira etapa de colheita. O tratamento com GA $550 \mathrm{ppm}$ promoveu aumento significativo de produção na segunda etapa, com relação às demais.

Na tabela 3 observamos que o número de frutos coletados mostrou-se inferior na primeira etapa de colheita. Análise isolada dos tratamentos comprovou este resultado geral, sendo que o tratamento com GA 550 ppm mostrou aumentar o número de frutos na segunda etapa e reduzir progressivamente na terceira e quarta etapas.

A tabela 3 mostra que o peso médio dos frutos revelou-se superior na primeira etapa, reduzindo-se significativamente, de acordo com o teste de Tukey, nas etapas subseqüentes de colheita. Analisando-se isoladamente o efeito dos tratamentos nas etapas de colheita, notamos este decréscimo no peso médio, da primeira até a quarta etapa, em todos os tratamentos.

$\mathrm{Na}$ tabela 4 observamos os resultados das análises de regressão de cada tratamento ( $\mathrm{T} 1$ a $\mathrm{T} 6$ ) sobre quatro etapas de colheita, para os parâmetros estudados. De acordo com a significância podemos auferir as curvas características da regressão linear (RL), regressão quadrática (RQ) e regressão cúbica (RC). Observou-se que o tratamento com GA 550 ppm promoveu aumento no comprimento dos pecíolos, estolhos e pedúnculos. Aplicação de IAA $10 \mathrm{ppm}$ ou GA 10 ppm, em 3 ocasiões, causou aumento sensível no comprimento dos estolhos e pedúnculos.

\section{DISCUSSÃO}

Verificou-se no presente ensaio que GA 550 ppm, SADH 5000 ppm e CCC 2000 ppm promoveram redução no peso dos frutos colhidos. Diminuição na produção do morangueiro, também foi observada por TAVAOZE \& MAZANASHVILI (1973), com aplicação de GA. Redução na colheita foi verificada com aplicação de SADH em concentrações acima de 2000 ppm (BERGAMINI \& PIMPINI, 1968), sendo que o SADH pode também promover aumentos na produtividade sob condições de estresse (PUFFER et alii, 1968; FREEMAN \& CARNE, 1970). CCC $3 \%$ promoveu aumentos na produtividade, sendo que CCC $5 \%$ pode mostrar efeitos depressivos na produção (GUERRIERO et alii, 1969; LORETI \& VITAGLIANO, 1970).

No presente ensaio IAA 10 ppm e GA 10 ppm, em 3 aplicações, mostraram uma tendência de aumentar a produção. SMITH et alii 
(1961) notaram aumento na produtividade do morangueiro cultivar "Sparkle" com 3 aplicações de GA 10 ppm.

Os reguladores de crescimento não afetaram o número de frutos do morangueiro; sendo que SADH $5000 \mathrm{ppm}$ atrasou a frutificação e GA $550 \mathrm{ppm}$ promoveu precocidade de maturação. Verificou-se que apesar de PUFFER et alii (1968) considerarem que o SADH pode afetar a precocidade de maturação dos frutos de morangueiro, BERGANINI \& PIMPINI (1968) observaram que o SADH não alterou a época de maturação. PATTLAK \& SINGH (1971) notaram aumento no número de frutos por planta com aplicação de GA. Precocidade na maturação dos frutos do morangueiro com aplicação de GA, também foi observada por SMITH et alii (1961), PATTLAK \& SINGH (1971) e HONDA (1972).

Aplicação de GA $550 \cdot \mathrm{ppm}$ reduziu o peso médio do fruto de morangueiro.

Na primeira etapa de colheita teve-se a menor produção de frutos, sendo que a maior produção foi observada na terceira etapa de colheita. Aumentos em produtividade na terceira etapa, foram obtidos com 3 aplicações de IAA $10 \mathrm{ppm}$ ou GA $10 \mathrm{ppm}$. SMITH et alii (1961) observaram aumentos em produção nas três primeiras colheitas, tendo notado diminuição no peso dos frutos em colheitas posteriores; sendo que 3 aplicações de GA $10 \mathrm{ppm}$ não afetaram a produção total do morangueiro.

Na primeira etapa de colheita teve-se o menor número de frutos; sendo que GA $550 \mathrm{ppm}$ aumentou o número de frutos na segunda etapa, e reduziu progressivamente na terceira e quarta etapas de colheita.

O peso médio dos frutos foi sempre superior nas primeiras colheitas (primeira etapa), reduzindo-se nas etapas subseqüentes.

Aumento no comprimento dos pecíolos, estolhos e pedúnculos foi obtido com GA $550 \mathrm{ppm}$. Resultados semelhantes foram observados por PATTLAK \& SINGH (1971) e por TAVAOZE \& MAZANASHVILI (1973). Pulverizações com IAA $10 \mathrm{ppm}$ e GA $10 \mathrm{ppm}$ aumentaram o comprimento dos pedúnculos, facilitando a colheita.

\section{CONCLUSÕES}

Os resultados obtidos neste ensaio, permitem as seguintes conclusões:

1. Apesar da aplicação de GA 550 ppm, SADH 5000 ppm e CCC $2000 \mathrm{ppm}$ reduzir a produção do morangueiro, três aplicações de IAA $10 \mathrm{ppm}$ ou GA $10 \mathrm{ppm}$ promovem uma tendência de aumento na produtividade da cultivar "Monte Alegre". 
2. Os reguladores de crescimento: $\mathrm{SADH}, \mathrm{CCC}$, IAA e GA, não alteram o número de frutos do morangueiro.

3. Aplicação de GA 550 ppm promove redução no peso médio dos frutos de morangueiro.

4. A menor produção do morangueiro concentra-se nas primeiras duas semanas de colheita; sendo que a maior produtividade verifica-se na quinta e sexta semanas de colheita.

5. O menor número de frutos verifica-se nas duas primeiras semanas de colheita; sendo que aplicação de GA $550 \mathrm{ppm}$ causa aumento no número de frutos na terceira e quarta semanas de colheita, reduzindo progressivamente nas semanas subseqüentes.

6. O peso médio dos frutos do morangueiro é maior nas primeiras duas semanas de colheita, reduzindo-se nas semanas seguintes.

7. Três aplicações de IAA $10 \mathrm{ppm}$ ou GA $10 \mathrm{ppm}$ promovem aumento no comprimento dos pedúnculos, facilitando a colheita.

\section{SUMMARY}

EFFECTS OF GROWTH RIEGULATORS ON THE FRUITING OF STRAWBERRY CULTIVAR “MONTE ALFGRI"

The effects of growth regulators on strawberry cultivar "Monte Alegre" were studied in Piracicaba, State of São Paulo.

Succinic acid -2,2 - dimethylhydrazide $\left(\mathrm{SADH}_{4}\right) 5000 \mathrm{ppm},(2-$ chloroethyl) trimethylammonium chloride (CCC) $2000 \mathrm{ppm}$, and gibberellic acid (GA) $550 \mathrm{ppm}$ were sprayed, and also three applications of indoleacetic acid (IAA) $10 \mathrm{ppm}$ or GA $10 \mathrm{ppm}$ were made. It was verified that although GA $550 \mathrm{pmm}$, SADH and CCC decreased yield, three applications of IAA $10 \mathrm{ppm}$ or GA $10 \mathrm{ppm}$ have promoted a tendency to increase strawberry yield. It was noted that growth regulators do not alter number of fruit, and GA $550 \mathrm{ppm}$ promotes reduction in fruit mean weight. GA ppm increased number of fruit on $3^{\text {rd }}$ and $4^{\text {th }}$ weeks of harvesting, decreasing progressively in subsequent weeks. It was observed that GA $10 \mathrm{ppm}$ or IAA $10 \mathrm{ppm}$ promotes an increase in peduncle length, easing harvesting.

\section{LITERATURA CITADA}

BERGAMINI, A.; PIMPINI, F. 1968. The effects of treatment with B- 9 during flowering on the strawberry cultivar Madame Moutot. Riv. Ortoflorofruttic. 52: 299-308.

COMARGO, L.S.; SCARANARI, H.J.; IGUE, T. 1974. Ensaio de cultivares e hibridos de morangueiro, Jundiaí, 1971. Bragantia 33(4) : 33-42. 
DENNIS, F.G.; BENNETT, H.O. 1969. Effect of gibberellic acid and deflowering upon runner and inflorescence development in an evergreen strawberry. Jour. Amer. Soc. Hort. Sci. 94: 534-537.

FREEMAN, J.A.; CARNE, I.C. 1970. Use of succinic acid 2,2 - dimethyl hydrazide (Alar) to reduce winter injury in strawberries. Canadian Jour. Plant Sci. 50: 189-190.

GUERRIERO, R.; LORETI, F.; VITAGLIANO, C. 1969. The effects of some growth regulators on the vegetative and reproductive behaviour of three strawberry cultivars. Riv. Ortoflorof ruttic. 53: 581-593.

HONDA, F. 1972. The effect of gibberellic acid on growth and flowering in strawberries. Bul. Hort. Res. Sta. Kurume $7: 45-57$.

LORETI, F.; VITAGLIANO, C. 1970. Further observations on the stimulatory effects of certain growth regulators on the productivity of strawberry plants. Riv. Ortoflorofrutic. $54: 547-555$.

PATTLAK, R.K.; SINGH, R. 1971. Effect of some external factors on the growth and fruiting of strawberry. II. Effect of GA, growth retardants and cloching on flowering and yield. Progr. Hort. 3(3): 53-63.

PUFFER, R.E.; VOTH, V.; BOWEN JR.; GRIPP, R.H. 1968 . Effects of Alar and top removal on yield of Fresno strawberries at three digging dates. Calif. Agric. 22(2): 11-13.

SMITH C.R.; SOCZEK, Z.; COLLINS, W.B. 1961. Flowering and fruting of strawberries in relation to gibberellins. Adv. Chem. Ser. 28: 109-115.

SOLOVEI, E.P. 1972. The effect of gibberellin on the development of vegetative organs in the strawberry. Akad. K. A. Timiryazeva 173: 35-38.

TAVAOZE, P.G.; MAZANASHVILI, T.G. 1973. The effect of gibberellic acid on. the growth and yield of large - fruited strawberries. Akad. Nauk Gruz 5: 13-15.

WEAVER, R.J. 1972. Plant growth substances in agriculture. San Francisco, W. H. Freeman. 594 pp. 
